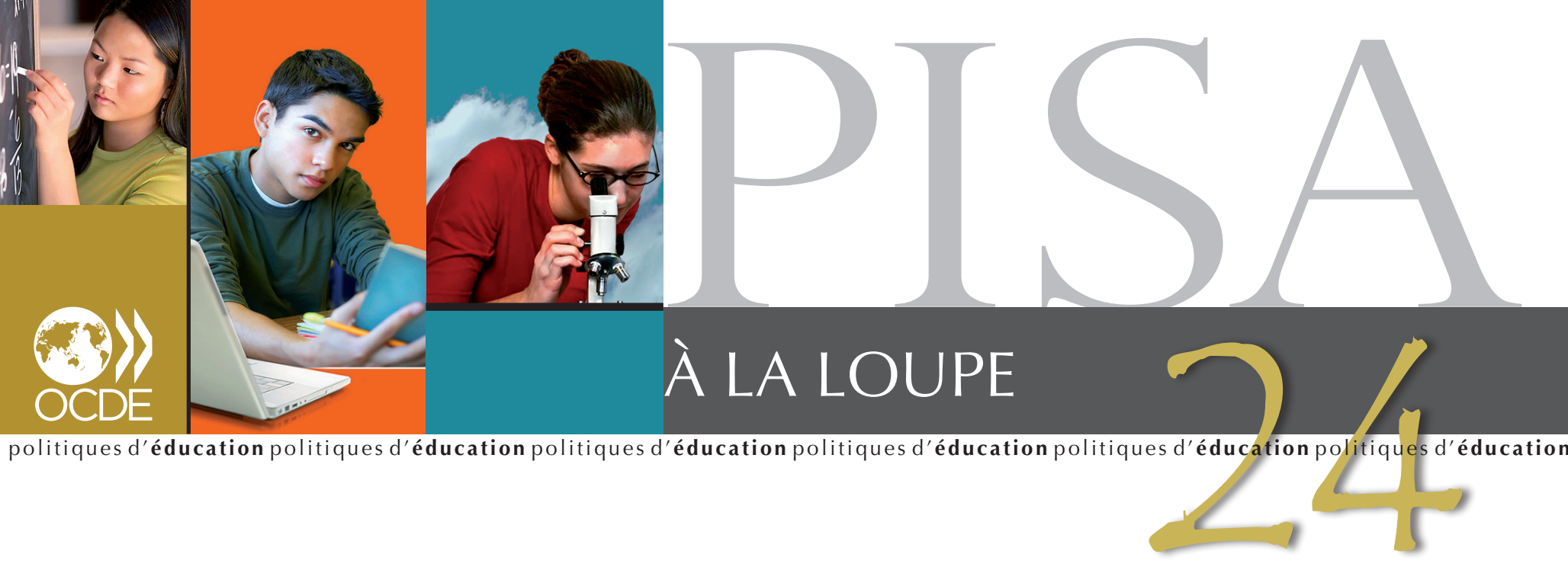

\title{
L'école : qu'en pensent les élèves ?
}

- La plupart des élèves pensent que ce qu'ils apprennent à l'école est utile pour eux-mêmes et pour leur avenir.

- Les attitudes des élèves envers l'école présentent une corrélation avec leurs compétences en compréhension de l'écrit.

- Les élèves qui déclarent que l'environnement de leur établissement est propice à l'apprentissage ont tendance à faire état d'attitudes plus positives envers l'école.

Les attitudes des élèves vis-à-vis de l'école peuvent être influencées par leurs enseignants, leurs camarades de classe ou encore par l'ambiance qui règne dans I'établissement. L'enquête PISA 2009 a cherché à déterminer si les élèves de 15 ans considèrent que ce qu'ils apprennent à l'école est utile, à la fois dans l'immédiat mais aussi pour leur avenir. Les questionnaires PISA ont demandé aux élèves s'ils pensaient que l'école ne les a pas beaucoup aidés à se préparer à la vie d'adulte au moment de quitter la scolarité ; qu'elle a été une perte de temps ; qu'elle les a aidés à gagner en confiance et à savoir prendre des décisions; et qu'elle leur a appris des choses utiles pour exercer un métier. Les élèves devaient indiquer s'ils n'étaient pas du tout d'accord, pas d'accord, d'accord ou tout à fait d'accord avec ces affirmations.

La plupart des élèves pensent

Dans les pays de l'OCDE, environ neuf élèves sur dix que l'école est utile... ont déclaré qu'ils ne pensaient pas que l'école avait été une perte de temps $(91 \%)$ et qu'ils pensaient que l'école leur avait enseigné des choses utiles pour exercer un métier ( $88 \%$ ). Quelque $76 \%$ des élèves pensaient que l'école les avait préparés à la vie d'adulte, et $74 \%$ d'entre eux étaient d'avis que l'école leur avait donné confiance dans leurs prises de décision.

Si, en général, les élèves font état d'attitudes positives envers l'école, leurs perceptions peuvent varier considérablement d'un pays à l'autre. Par exemple, en Albanie, en Indonésie, au Kazakhstan, au Mexique, en Thaïlande, à Trinité et Tobago et en Tunisie, plus de $90 \%$ des élèves pensent que l'école les a aidés à gagner en confiance pour prendre des décisions, tandis qu'en Allemagne, en Corée, au Japon, au Liechtenstein et au Luxembourg, moins de $60 \%$ des élèves sont de cet avis. Toutefois, les élèves de différents pays ne veulent peut-être pas toujours dire la même chose lorsqu'ils répondent à ces questions. 


\section{Les élèves pensent-ils que l'école est utile ?}

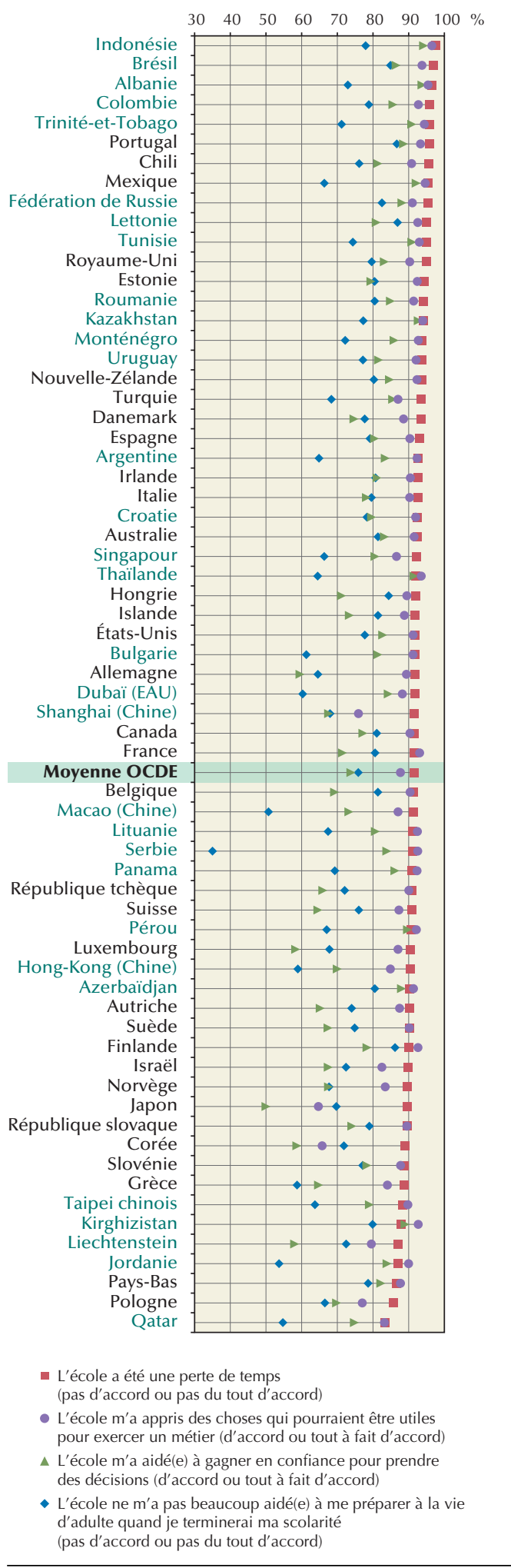

... et les élèves qui pensent que l'école est utile ont tendance à obtenir de bons résultats...

Dans la plupart des pays, les élèves qui pensent que l'école est utile sont davantage susceptibles d'obtenir un score élevé à l'évaluation PISA de la compréhension de l'écrit, et les élèves qui obtiennent un score élevé en compréhension de l'écrit ont tendance à déclarer qu'ils pensent que l'école est utile. Dans 48 pays et économies, les élèves qui ont obtenu de bons résultats en compréhension de l'écrit ont tendance à faire état d'attitudes plus positives envers l'école que ceux qui ont obtenu des scores inférieurs. Ce n'est qu'en Allemagne, en Grèce et à Shanghai (Chine) qu'on a relevé une corrélation faible mais négative ; cependant, en Allemagne et en Grèce, la plus grande part de cette corrélation s'explique par des caractéristiques contextuelles des élèves et des écoles.

... et ont tendance à entretenir de bonnes relations avec leurs enseignants et à étudier dans des environnements propices à l'apprentissage.

Dans tous les pays et économies participants, les attitudes positives des élèves envers l'école sont liées à des attitudes positives envers leurs enseignants. Les élèves qui ont déclaré qu'ils s'entendaient bien avec leurs enseignants, que la plupart de leurs enseignants s'intéressaient à leur bien-être, que la plupart de leurs enseignants écoutaient ce qu'ils avaient à dire, que s'ils avaient besoin d'aide, leurs enseignants leur offraient cette aide, et que la plupart de leurs enseignants les traitaient de façon juste, avaient également tendance à déclarer que ce qu'ils apprennent à l'école est utile. Même après prise en compte de diverses caractéristiques au niveau des élèves et des établissements, cette relation positive s'observe dans tous les pays et économies participants.

La perception par les élèves de l'existence d'un environnement propice à l'apprentissage dans leur classe est aussi liée à leurs attitudes envers l'école. Les élèves qui ont déclaré que, pendant les cours, leurs camarades de classe n'écoutent pas l'enseignant, qu'il y a du bruit et de l'agitation, que l'enseignant doit attendre un long moment avant que les élèves ne se calment, que les élèves ne peuvent pas bien travailler, et qu'ils ne commencent à travailler que bien après le début du cours, ont tendance à penser que l'école n'est pas utile pour eux-mêmes et pour leur avenir. Il convient de noter que cette relation ne reflète pas uniquement le milieu social : à part au Liechtenstein, la corrélation apparaît clairement, même après prise en compte des caractéristiques au niveau des élèves et des établissements. 


\section{Qui est davantage susceptible de considérer que l'école est utile?}

\begin{tabular}{|c|c|c|c|c|c|c|c|c|}
\hline \multicolumn{9}{|l|}{ Australie } \\
\hline \multirow{2}{*}{\multicolumn{9}{|c|}{ Autriche }} \\
\hline \multicolumn{5}{|l|}{ Belgique } & & & & \\
\hline \multicolumn{9}{|l|}{ Canada } \\
\hline \multicolumn{9}{|l|}{ Chili } \\
\hline \multirow{2}{*}{\multicolumn{9}{|c|}{ République tchèque }} \\
\hline \multicolumn{6}{|l|}{ Danemark } & & & \\
\hline \multirow{2}{*}{\multicolumn{9}{|c|}{ Estonie }} \\
\hline \multirow{2}{*}{\multicolumn{5}{|c|}{$\begin{array}{l}\text { Finlande } \\
\text { France }\end{array}$}} & & & & \\
\hline \multirow{2}{*}{\multicolumn{9}{|c|}{ Allemagne }} \\
\hline & & & & & & & & \\
\hline \multirow{2}{*}{\multicolumn{9}{|c|}{ Grèce }} \\
\hline & & & & & & & & \\
\hline Islande & & & & & & & & \\
\hline Irlande & & & & & & & & \\
\hline Israël & & & & & & & & \\
\hline Italie & & & & & & & & \\
\hline Japon & & & & & & & & \\
\hline Corée & & & & & & & & \\
\hline Luxembourg & & & & & & & & \\
\hline Mexique & & & & & & & & \\
\hline Pays-Bas & & & & & & & & \\
\hline Nouvelle-Zélande & & & & & & & & \\
\hline Norvège & & & & & & & & \\
\hline Pologne & & & & & & & & \\
\hline Portugal & & & & & & & & \\
\hline République slovaque & & & & & & & & \\
\hline Slovénie & & & & & & & & \\
\hline Espagne & & & & & & & & \\
\hline Suède & & & & & & & & \\
\hline Suisse & & & & & & & & \\
\hline Turquie & & & & & & & & \\
\hline Royaume-Uni & & & & & & & & \\
\hline États-Unis & & & & & & & & \\
\hline Albanie & & & & & & & & \\
\hline Argentine & & & & & & & & \\
\hline Azerbaïdjan & & & & & & & & \\
\hline Brésil & & & & & & & & \\
\hline Bulgarie & & & & & & & & \\
\hline Colombie & & & & & & & & \\
\hline Croatie & & & & & & & & \\
\hline Dubaï (EAU) & & & & & & & & \\
\hline Hong-Kong (Chine) & & & & & & & & \\
\hline Indonésie & & & & & & & & \\
\hline Jordanie & & & & & & & & \\
\hline Kazakhstan & & & & & & & & \\
\hline Kirghizistan & & & & & & & & \\
\hline Lettonie & & & & & & & & \\
\hline Liechtenstein & & & & & & & & \\
\hline Lituanie & & & & & & & & \\
\hline Macao (Chine) & & & & & & & & \\
\hline Monténégro & & & & & & & & \\
\hline Panama & & & & & & & & \\
\hline Pérou & & & & & & & & \\
\hline Qatar & & & & & & & & \\
\hline Roumanie & & & & & & & & \\
\hline Fédération de Russie & & & & & & & & \\
\hline Serbie & & & & & & & & \\
\hline Shanghai (Chine) & & & & & & & & \\
\hline Singapour & & & & & & & & \\
\hline Taipei chinois & & & & & & & & \\
\hline Thaïlande & & & & & & & & \\
\hline Trinité-et-Tobago & & & & & & & & \\
\hline Tunisie & & & & & & & & \\
\hline Uruguay & & & & & & & & \\
\hline Résumé pour & $\begin{array}{l}\text { Relation } \\
\text { positive } \\
65 / 65\end{array}$ & $\begin{array}{l}\text { Plus } \\
\text { propice } \\
64 / 65\end{array}$ & $\begin{array}{l}\text { Filles } \\
28 / 65\end{array}$ & $\begin{array}{l}\text { Milieu } \\
\text { favorisé } \\
21 / 65\end{array}$ & $\begin{array}{c}\text { Élèves issus de } \\
\text { l'immigration } \\
18 / 49\end{array}$ & $\begin{array}{c}\text { Établissement } \\
\text { privé } \\
9 / 49\end{array}$ & $\begin{array}{c}\text { Grand } \\
\text { établissement } \\
13 / 64\end{array}$ & $\begin{array}{c}\text { Filière } \\
\text { professionnelle } \\
13 / 40\end{array}$ \\
\hline tous les participants & $\begin{array}{l}\text { Relation } \\
\text { négative } \\
0 / 65\end{array}$ & $\begin{array}{l}\text { Moins } \\
\text { propice } \\
0 / 65\end{array}$ & $\begin{array}{c}\text { Garcons } \\
5 / 65\end{array}$ & $\begin{array}{c}\text { Milieu } \\
\text { défavorisé } \\
9 / 65\end{array}$ & $\begin{array}{c}\text { Élèves } \\
\text { autochtones } \\
4 / 49\end{array}$ & $\begin{array}{c}\text { Établissement } \\
\text { public } \\
0 / 49\end{array}$ & $\begin{array}{c}\text { Petit } \\
\text { établissement } \\
2 / 64\end{array}$ & $\begin{array}{c}\text { Filière } \\
\text { générale } \\
3 / 40\end{array}$ \\
\hline
\end{tabular}

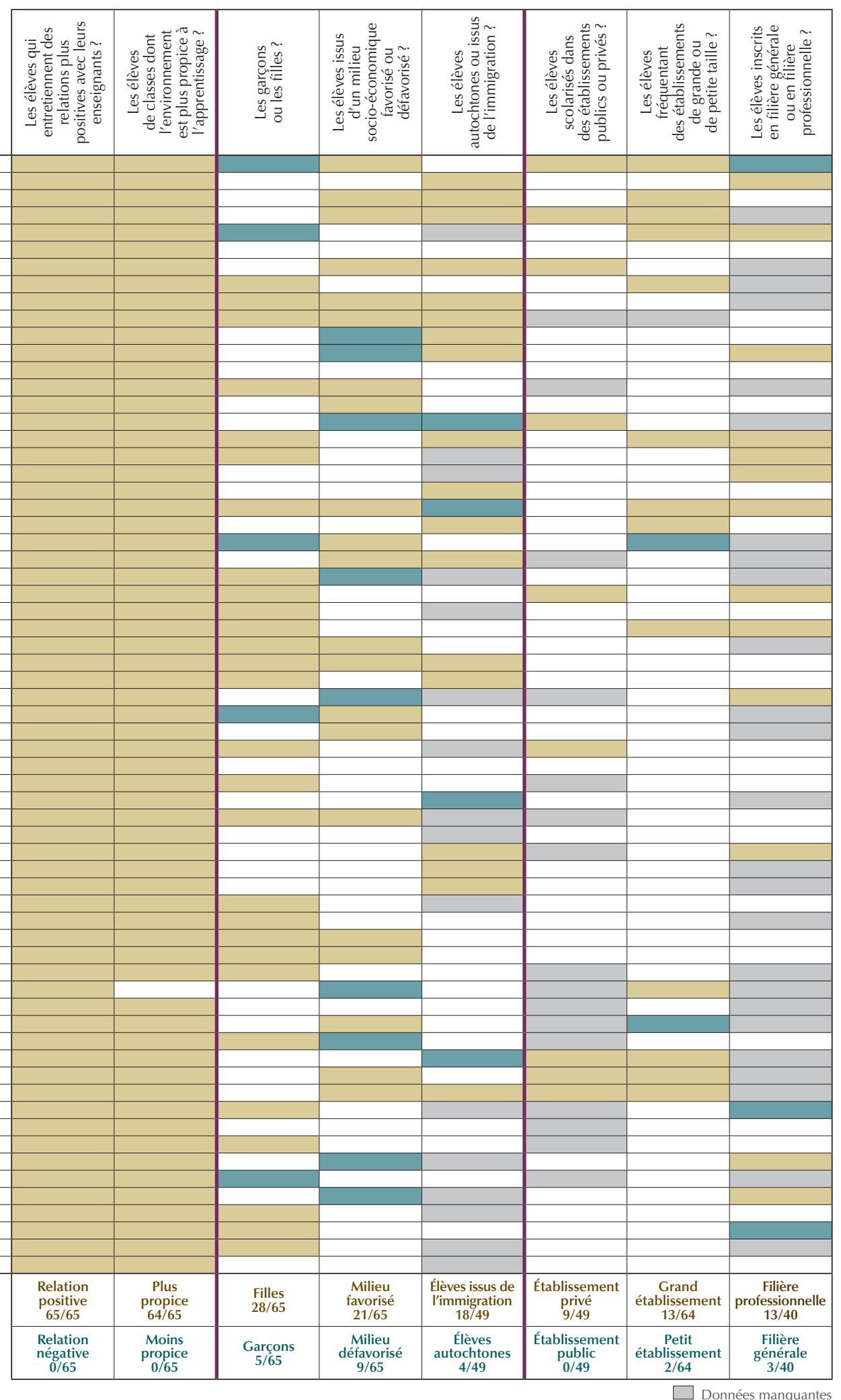

Remarque : les relations entre les attitudes des élèves envers l'école et différentes caractéristiques au niveau des élèves (sexe, milieu socio-économique et statut au regard de l'immigration) et au niveau des établissements (climat, type d'établissement, taille, orientation des programmes scolaires et situation géographique) sont considérées ensemble. Les attitudes des élèves envers l'école sont mesurées par un indicateur composite prenant en compte ces quatre questions. 
En réalité, ces associations se renforcent mutuellement. Les élèves qui entretiennent de bons rapports avec leurs enseignants et qui étudient dans un environnement propice à l'apprentissage auront tendance à penser que l'école est utile - et leurs attitudes positives améliorent le climat à l'école.

Les attitudes des élèves envers l'école ne sont que faiblement liées à leur propre milieu ou aux types d'établissements qu'ils fréquentent.

Dans 28 des 65 pays et économies participants, les filles ont tendance à faire état d'attitudes plus positives envers l'école que les garçons, lorsque le climat à l'école et les caractéristiques contextuelles des élèves et des établissements sont pris en compte. Ce n'est qu'en Australie, au Chili, en Nouvelle-Zélande, au Royaume-Uni et à Singapour que les garçons ont tendance à faire état d'attitudes plus positives envers l'école que les filles. Dans 21 pays et économies participants, les élèves issus de milieux socio-économiques favorisés ont tendance à faire état d'attitudes plus positives envers l'école, tandis qu'on constate l'inverse dans neuf pays et économies. Dans 18 des 49 pays et économies dont les données sont comparables, les élèves issus de l'immigration ont tendance à faire état d'attitudes plus positives envers l'école que les élèves autochtones. La situation inverse s'observe uniquement au Brésil, en Israël, en Lituanie, au Mexique et au Panama.

Dans 9 des 49 pays et économies dont les données sont comparables, les élèves fréquentant des établissements privés ont tendance à faire état d'attitudes plus positives envers l'école que les élèves des établissements publics.
S'agissant de l'association entre la taille des établissements et l'orientation des programmes scolaires d'un établissement, d'une part, et les attitudes des élèves envers l'école, d'autre part, aucune corrélation systématique ne se dégage. Dans 13 pays, les élèves scolarisés dans des établissements plus grands ont tendance à faire état d'attitudes plus positives envers l'école que les élèves fréquentant des établissements plus petits, mais on constate l'inverse dans deux pays. Dans 13 des 40 pays et économies dont les données sont comparables, les élèves scolarisés en filière professionnelle ont tendance à faire état d'attitudes plus positives envers l'école que les élèves scolarisés en filière générale, mais on observe l'inverse dans trois pays.

Les caractéristiques contextuelles des élèves et des établissements ne présentent qu'un lien ténu avec l'attitude des élèves envers l'école, tandis que le climat de l'établissement y est fortement corrélé. Parmi les pays de l'OCDE, lorsque différentes caractéristiques contextuelles des élèves (sexe, milieu socio-économique et situation au regard de l'immigration) et des établissements (type d'établissement, taille, orientation des programmes et situation géographique) sont toutes prises en compte, seuls $2 \%$ de la variation totale des attitudes des élèves envers l'école s'expliquent. En revanche, la prise en compte du climat de l'établissement (c'est-à-dire les relations élèves-enseignants et le climat de discipline) permet d'expliquer quelque $20 \%$ de la variation des attitudes des élèves envers l'école.

Pour conclure: Les attitudes des élèves envers l'école et leur performance en compréhension de l'écrit se renforcent mutuellement, tout comme leurs attitudes envers l'école et le climat d'apprentissage en classe. Cela signifie que, dans une certaine mesure, ce sont les attitudes des élèves eux-mêmes qui peuvent façonner leur expérience individuelle d’apprentissage.

Pout tout complément d'information

Contacter Miyako Ikeda (Miyako.lkeda@oecd.org)

Consulter Données sous-jacentes

Voir

www.pisa.oecd.org

www.oecd.org/pisa/infocus
Prochain numéro

Les pays s'orientent-ils vers des systèmes d'éducation plus équitables? 